\title{
Synthesis of metal matrix nano composite for better ware and damping in corrosive environment
}

\author{
B. S. Ben ${ }^{1}$, G. Raghavendra ${ }^{1}$, Syed Ismail ${ }^{1}$ and B. A. Ben ${ }^{2}$. \\ ${ }^{1}$ Department of mechanical engineering, NIT Warangal, India, 506004 \\ ${ }^{2}$ Department of mechanical engineering, VIGNAN Institute of information technology, Vizag, India, \\ 530049 \\ *Email: satishben@nitw.ac.in
}

A metal matrix composite (MMC) has been synthesized with granite dust as reinforcement using vortex method. The aim of the present work is to investigate the enhanced damping and wear resistance characteristics of synthesized composite material. A2024/granite composites were fabricated with 5, 10, and 15 weight percent (wt\%) reinforcement. The wear characterization was carried out using Pin-on-disc wear testing machine (DUCOM TL) with data acquisition system. Dynamic Mechanical Analysis was carried out using Non-destructive evaluation (NDE) technique (Bandwidth method). The study of damping behaviour of the composites was carried out using ultrasonic pulse generator setup. The results of all the experiments show that the developed composites exhibit improved characteristics compared to unreinforced alloy.

The ceramic particulate reinforced composites exhibit improved abrasion resistance as well as good damping properties. They find applications as cylinder blocks, pistons, piston insert rings, brake disks and callipers [1]. The damping capacity of a material is the fundamental property for designing and manufacturing structural components in dynamic applications. The damping property is a measure of a material's ability to dissipate elastic strain energy during mechanical vibration or wave propagation [2]. This investigation aims at characterizing the wear and damping behavior in A2024-Granite dust composites processed by stir casting technique or vortex method [3]. Granite dust particles have been used in this work as discontinuous dispersoids for developing high damping and wear resistance MMC. Granite dust is having low-density with high damping capacity and it is also low-cost since available in large quantities as a waste by-product in granite processing industries [4].

High energy ball milling was carried out using Retsch PM100 planetary ball mill with zirconium and tungsten carbide as milling media and keeping the ball to powder ratio as 10:1. Phase identification in the composites has been carried out by X-ray diffraction (XRD). The solid samples were prepared as solid pieces using a low speed saw, and then ground manually with a 1200 grit SiC paper. Then samples are polished on a polishing cloth on polishing machine with water as coolant/lubricant.

The initial average size of the granite powder has been measured as $5 \mu \mathrm{m}$ using XRD crystallographic analysis and then the granite powder was wet milled in a high energy ball mill for 5 hours. The average particle size (ASP) of the wet milled granite powder is measured to be $70 \mathrm{~nm}$ and similarly the synthesized nano powders are mechanical milled with aluminum alloy powder in different weight fractions. SEM photograph of a mechanically milled aluminum alloy powder with granite powder is shown in Figure 1a. Again, Figure 1b shows the wet milled granite powder analyzed by XRD machine and the phases were identified by the database provided by the software interface.

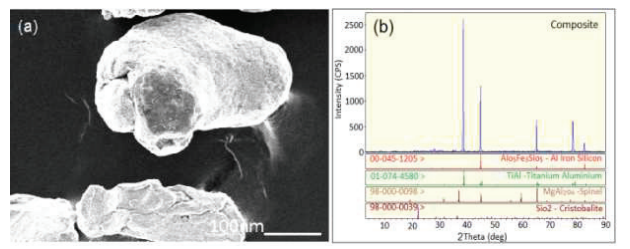

Figure 1: (a) SEM Image of granite particle penetrating into Matrix material (b) XRD analysis of high energy ball milled composite powder

The process graph for the preparation of the composite with highlighted process points is shown in Figure 2.

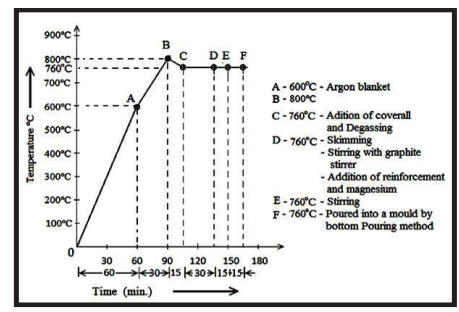

Figure 2: Process Graph for composite preparation

\section{References}

1. K.G. Rohatgi, Composites Science and Technology, 67, (2007),3369-3377

2. G.H. Wu, Z.Y Dou,. L.T Jiang, J.H Cao, Materials Letters. 60, (2006) 2945-2948

3. M. Kok, composites: part A. 37, (2006),457-464.

4. S.C Sharma, Wear. 249, (2001),1036-1045. 